\title{
Phase contrast tomography: An alternative approach
}

\author{
A. Groso, ${ }^{a}$ M. Stampanoni, and R. Abela \\ Swiss Light Source, Paul Scherrer Institut, CH-5232 Villigen PSI, Switzerland \\ P. Schneider, S. Linga, and R. Müller \\ Institute for Biomedical Engineering, Swiss Federal Institute of Technology (ETH) and University of Zürich, \\ Zürich, Switzerland
}

(Received 30 August 2005; accepted 28 April 2006; published online 25 May 2006)

\begin{abstract}
Conventional microtomography is widely used for reconstructing the three-dimensional (3D) distribution of the absorption coefficient of a sample from a set of radiographic projections. The question here is whether the 3D distribution of the phase (refractive index) of a weakly absorbing object can be, alternatively to the combined phase retrieval-backprojection methods presented up to now, directly reconstructed from a single tomographic data set. In this letter a solution to this problem based on a direct filtered backprojection approach, derived from the transport of intensity equation, is investigated numerically and experimentally for monochromatic and polychromatic $\mathrm{x}$ rays. The results show that the contrast is increased, while keeping dose minimal and spatial resolution equivalent to the conventional absorption based technique. This method is therefore very promising for imaging of biological specimens. (C) 2006 American Institute of Physics.
\end{abstract}

[DOI: $10.1063 / 1.2207221]$

Conventional x-ray computed tomography (CT) is based on the difference in $\mathrm{x}$-ray absorption by different materials and is nowadays a standard technique with spatial resolution around $1 \mu \mathrm{m} .{ }^{1}$ On the other hand, a wide range of samples studied in materials science, biology, and medicine shows a very weak absorption contrast, yet producing significant phase shifts of the x-ray beam. The use of phase information for imaging purposes is therefore a suitable alternative. Phase contrast imaging techniques have two key advantages: first, light elements-showing poor contrast in absorption radiography — can be easily detected and second, phase contrast radiography helps to reduce the radiation dose deposited on the object under investigation.

As far as phase tomography [quantitative threedimensional (3D) reconstruction of the phase or the refractive index from two-dimensional (2D) phase images] is concerned, several attempts were made by using interferometric $^{2,3}$ and noninterferometric phase retrieval methods. ${ }^{4-10}$ All these methods are based on a two-step approach: first, the projections of the phase are determined in the form of Radon projections and then the object function, i.e., the refractive index decrement $\delta$ is reconstructed by applying a conventional filtered backprojection algorithm. Since far from absorption edges $\delta$ is linearly related to the electron density $\rho$, which is-except for hydrogen rich materials - proportional to the mass density, the resulting reconstruction represents approximately the distribution of the mass density in the sample. ${ }^{11}$

Alternatively, Bronnikov suggested an algorithm ${ }^{12,13}$ which eliminates the intermediate step of $2 \mathrm{D}$ phase retrieval and provides a direct $3 \mathrm{D}$ reconstruction of the phase (index of refraction) of the object. In his approach and for the purpose of our own study, the scanning geometry is defined as follows (Fig. 1). The $x_{3}$ axis of the reference coordinate system $\left(x_{1}, x_{2}, x_{3}\right)$ defines the axis of rotation of the tomographic

${ }^{a}$ Author to whom correspondence should be addressed; electronic mail: amela.groso@psi.ch scan. The rotated coordinate system $(x, y, z)$ is related to the reference system by $x=x_{1} \cos \theta+x_{2} \sin \theta$ and $z=x_{2} \cos \theta$ $-x \sin \theta$, where the angle $\theta$ is measured from the positive $x_{1}$ axis and denotes the orientation of the two-dimensional intensity projection, which is measured by a detector parallel to the $(x, y)$ plane and which is located at distance $z=d$. Consider a monochromatic plane wave $U_{\text {inc }}$ with wavelength $\lambda$ that propagates along the positive $z$ axis and that impinges upon a thin mixed phase-amplitude object, which is characterized by the linear absorption coefficient $\mu\left(x_{1}, x_{2}, x_{3}\right)$ and the real part of the index of refraction $\delta\left(x_{1}, x_{2}, x_{3}\right)$.

With $I_{\theta, z=0}(x, y)=\left|\exp \left[-(1 / 2) \mu_{\theta}(x, y)\right] U_{\text {inc }}\right|^{2}$ being the absorption contrast intensity measured at a sample-todetector distance (SDD) $z=0$, and

$$
\begin{aligned}
\phi_{\theta}(x, y)= & -\frac{2 \pi}{\lambda} \int_{\mathfrak{R}^{2}}\left[\delta\left(x_{1}, x_{2}, y\right)\right. \\
& \left.\times \tilde{\delta}\left(x-x_{1} \cos \theta-x_{2} \sin \theta\right)\right] d x_{1} d x_{2}
\end{aligned}
$$

being the phase function of the object (the Dirac function is denoted by $\tilde{\delta}$ in order to differentiate from the refractive index $\delta$ ), the intensity distribution at distance $z=d$ and angle of rotation $\theta$ is approximated by the following expression: ${ }^{14}$

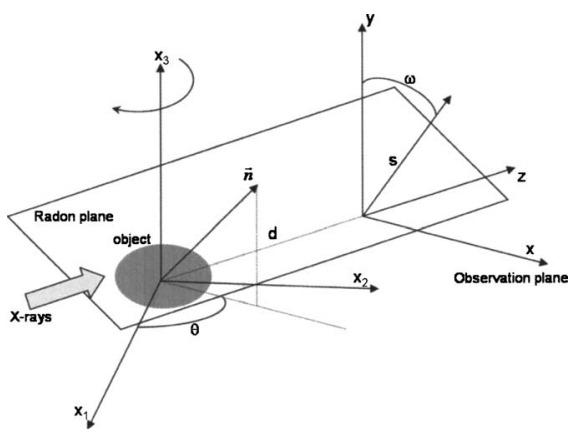

FIG. 1. Tomographic scanning geometry adapted from Ref. 13. 


$$
I_{\theta, z=d}(x, y)=I_{\theta, z=0}(x, y)\left[1-\frac{\lambda d}{2 \pi} \nabla^{2} \phi_{\theta}(x, y)\right],
$$

with $\nabla^{2} \equiv\left(\partial^{2} / \partial x^{2}\right)+\left(\partial^{2} / \partial y^{2}\right)$. This is valid in the near field Fresnel region, where $d \ll a^{2} / \lambda$ ( $a$ is the transversal size of the smallest structure in the object and $\lambda$ is the $\mathrm{x}$-ray wavelength), and for a mixed phase-amplitude object with weak and almost homogeneous absorption, i.e., $\partial \mu_{\theta} / \partial x, \partial \mu_{\theta} / \partial y$ $\approx 0$. For a pure phase object Eq. (2), which also corresponds to the transport of intensity equation (TIE) in its simplified form, ${ }^{6-9}$ can further be expressed as

$$
I_{\theta, z=d}(x, y)=1-\frac{\lambda d}{2 \pi} \nabla^{2} \phi_{\theta}(x, y) .
$$

The goal of quantitative phase tomography is to reformulate Eq. (2) to obtain $\delta\left(x_{1}, x_{2}, x_{3}\right)$ from the knowledge of $I_{\theta, z=d}(x, y)$ for $\theta \in[0, \pi]$. Expression (2) can also be written as $\quad \nabla^{2} \phi_{\theta}(x, y)=-(2 \pi / \lambda d) g_{\theta}(x, y)$, where $g_{\theta}(x, y)$ $=\left[I_{\theta, z=d}(x, y)\right] /\left[I_{\theta, z=0}(x, y)\right]-1$ denotes a data function that is completely determined from the known quantities $I_{\theta, z=d}(x, y)$ and $I_{\theta, z=0}(x, y)$.

The 2D Radon transform $\hat{g}(s, \omega)=\int_{\mathfrak{R}^{2}} g(x, y)$ $\times \tilde{\delta}(s-x \sin \omega-y \cos \omega) d x d y$ describes the integration of the function $g(x, y)$ over the lines given by the equation $s$ $=x \sin \omega+y \cos \omega$ in the $x y$ plane, whereas the 3D Radon transform $\quad \hat{\delta}(s, \theta, \omega)=\int_{\mathfrak{R}^{3}} \delta\left(x_{1}, x_{2}, x_{3}\right) \times \tilde{\delta}\left(s-\left(x_{1} \cos \theta\right.\right.$ $\left.\left.+x_{2} \sin \theta\right) \times \sin \omega-x_{3} \cos \omega\right) d x_{1} d x_{2} d x_{3}$ of the function $\delta\left(x_{1}, x_{2}, x_{3}\right)$ describes the integration over planes rather than lines. Such a plane is perpendicular to the unit vector $n$ $=(\cos \theta \sin \omega, \sin \theta \sin \omega, \cos \omega)$ and is at distance $s$ from the origin.

Applying the Radon transform (denoted by the symbol ${ }^{\wedge}$ ) to Eq. (1) and calculating the second derivative with respect to the variable $s=x \sin \omega+y \cos \omega$ one gets ${ }^{13}$

$$
\frac{\partial^{2}}{\partial s^{2}} \hat{\delta}(s, \theta, \omega)=-\frac{1}{d} \hat{g_{\theta}}(s, \omega) .
$$

Expression (3) is a theorem which states that from the 2D Radon transform of the measured value $g$, one can directly find the 3D Radon transform of $\delta$. Considering that an explicit inversion formula for the 3D Radon transform was given by Radon and Lorentz already at the beginning of the previous century, one has a direct solution to the reconstruction problem for quantitative phase contrast CT, namely, $\delta\left(x_{1}, x_{2}, x_{3}\right)=-\left(1 / 4 \pi^{2} d\right) \int_{0}^{\pi} \sin \omega d \omega \int_{0}^{\pi} d \theta \hat{g}_{\theta}\left(s^{\prime}, \omega\right)$. The computation of the two-dimensional Radon transform and backprojection over the angle $\omega$ can be combined in a single step of the algorithm. Namely, inserting the equation for the 2D Radon transform of the data $g_{\theta}$, calculating the integral over the angle $\omega$, and defining $q(x, y)=|y| /\left(x^{2}+y^{2}\right)$ one obtains ${ }^{13}$

$$
\delta\left(x_{1}, x_{2}, x_{3}\right)=\frac{1}{4 \pi^{2} d} \int_{0}^{\pi}\left(q * * g_{\theta}\right) d \theta
$$

where the stars indicate a 2D convolution. Expressed in this (filtered backprojection) form this approach becomes very interesting since in the case of a pure phase object [i.e., $\left.I_{\theta, z=0}(x, y)=1\right]$ it allows recovering the refractive index in $3 \mathrm{D}$ from only one single tomographic data set: $g_{\theta}(x, y)$ $=I_{\theta, z=d}(x, y)-1, \theta \in[0, \pi]$. This is a significant step since (a) the experimental setup can be kept extremely simple (it is

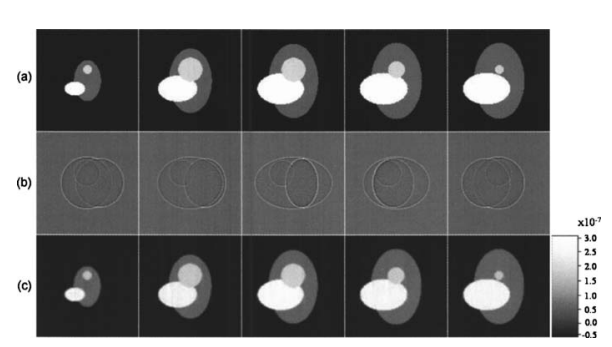

FIG. 2. Simulation results: (a) Cross-sectional views of the $3 \mathrm{D}$ object function $\delta$ with values of 0 (black), $1.3 \times 10^{-7}, 2.6 \times 10^{-7}$, and $3 \times 10^{-7}$ (white). (b) Calculated intensity for five different projection angles $\theta$ at a sample-todetector distance of $25 \mathrm{~mm}$. (c) Cross-sectional view of the reconstructed $3 \mathrm{D}$ pure phase object with corresponding gray scale bar.

actually the same as for standard, absorption based tomography) and (b) the radiation damage is kept to the minimum, which is of special importance for biological specimens and for potential future in vivo studies. In addition, time consuming data alignment routines as well as phase retrieval processes become superfluous.

In order to validate the theory using a practical implementation, we first simulated intensity projection data and reconstructed the object according to the aforementioned theory. The object used for the simulation was a mathematical phantom made up of three spheres $(120,50$, and 30 voxel diameter) inside a cube [Fig. 2(a)]. Two cases, a pure phase object $[\mu=(4 \pi / \lambda) \beta=0$, where $\beta$ is the imaginary part of the refractive index] and mixed, weakly absorbing phase object ( $\beta$ of the order of $10^{-9}$ ) were studied. The values for the index of refraction $\delta$ were $2.6 \times 10^{-7}$ for the outermost sphere, $1.3 \times 10^{-7}$ and $3 \times 10^{-7}$ for the two smaller spheres, and 0 for the cube and they were kept the same for both pure phase and mixed object case. Cross sections of the simulated pure phase object are shown in Fig. 2(a). The phase function $\phi$ was calculated analytically from the knowledge of $\delta$ and sets of phase contrast projection data were generated using the Fresnel propagator ${ }^{15}$ with $\lambda=1 \AA$, SDD $=25 \mathrm{~mm}, 185^{2}$ detector array, and an isotropic pixel size of $1 \mu \mathrm{m}$. Five (out of 200) angular phase contrast projections corresponding to $\theta=0, \pi / 4, \pi / 2,3 \pi / 4, \pi$ are shown in Fig. 2(b). Using Eq. (4), the phase information (refractive index) has been successfully reconstructed with errors (compared to tabulated values) lying between $1 \%$ for the pure phase objects [Fig. $2(c)]$ and $6 \%$ for mixed phase-amplitude objects.

In a second step, the algorithm has been used to reconstruct phase tomograms from experimental data (projections), obtained at the Tomography Station of the Materials Science beam line of the Swiss Light Source. ${ }^{16}$ As presented in this work, to our knowledge this has been done for the first time for an inhomogeneous phase object using this integrative one-step approach. In order to cope with the more stringent requirements given by the experimental conditions (detector sensitivity and noise and beam instability) the original method has been amended by adding an ad hoc filtering process prior to backprojection step, leading to a modified version of Bronnikov's algorithm (MBA).

A sample consisting of a polyethylene tube with two polymer fibers and a human hair has been investigated at a wavelength of $0.91 \AA$, an isotropic pixel size of $1.75 \mu \mathrm{m}$, and a SDD of $150 \mathrm{~mm}$. The results are given in Fig. 3, which compares a reconstructed slice obtained using (a) conventional filtered backprojection (absorption contrast) and (b) the MBA (phase contrast). 

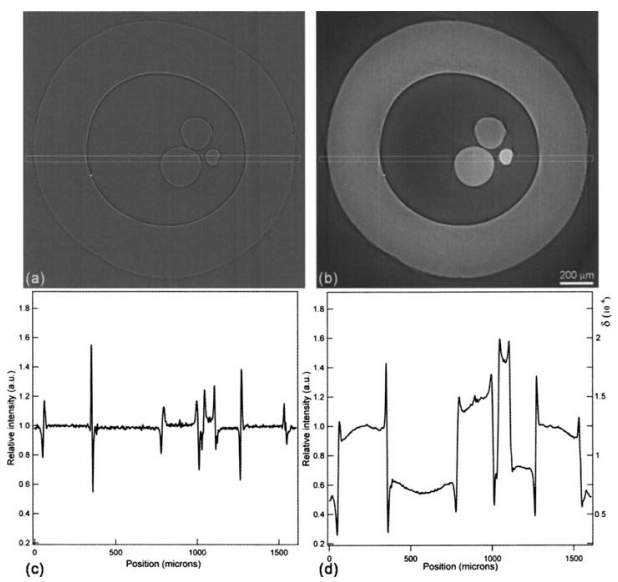

FIG. 3. Reconstructed slice of a polyethylene tube with polymer fibers inside. (a) Using conventional filtered backprojection and (b) using the modified Bronnikov algorithm (MBA). Bottom: Line profiles integrated through the sections indicated by rectangles in the tomograms (a) and (b). The intensity value corresponding to polyethylene was normalized to 1 in both tomograms. The scale on the right side of graph (d) gives the corresponding literature values (Ref. 17) for the real part of the refractive index.

The advantages of the MBA are evident: while in Fig. 3(a) only edges are enhanced but no information about the inside of the sample is obtained, in Fig. 3(b) the weakly absorbing materials can be clearly distinguished thanks to the difference in phase. Similar results were obtained for other test samples (data not shown). Additionally, if a suitable calibration is provided, the MBA delivers quantitative information within a $10 \%$ error, as shown in Fig. 3(d). This error is justified by the approximation we used, which does not take into account either detector noise (a few percent) or sample absorption contribution (also a few percent). Therefore, the reconstructed value for the refractive index of polyethylene was normalized to its tabulated record ${ }^{17}$ and the $\delta$ values of the other components scale within the aforementioned error range.

The edge enhancement effect visible in the reconstruction [Fig. 3(b)] can be explained by the combined influences of the additional filtering, interpolation in the backprojection step, and the fact that a SDD of $150 \mathrm{~mm}$ was not ideal for the near field Fresnel regime approximation.

In order to investigate the robustness of the MBA with respect to energy resolution a test sample (polystyrene spheres glued together) has been scanned using the so-called pink beam mode. After the removal of the monochromator, the upper part of the spectrum has been cut at $18 \mathrm{keV}$ (using $\mathrm{Rh}$ mirrors) while the lower part has been suppressed by a $75 \mu \mathrm{m}$ thick $\mathrm{Zr}$ filter. In addition, the absorption edge of the YAG:Ce scintillator $(17.4 \mathrm{keV})$ has been exploited to define the energy around $17.5 \pm 0.5 \mathrm{keV}$. Figures 4(a)-4(c) present

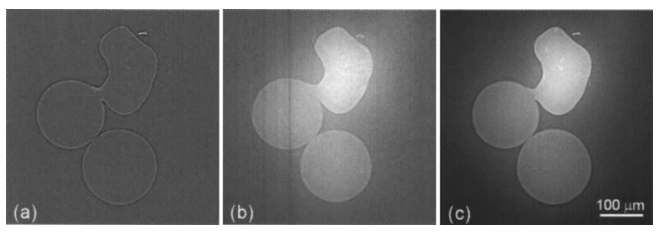

FIG. 4. Polystyrene spheres reconstructed using (a) filtered backprojection, using MBA applied to (b) data taken with a narrow $0.014 \%$ energy bandwidth (monochromatic) and (c) data taken with a relatively broad 5\% bandwidth (polychromatic). results obtained using conventional filtered backprojection, using MBA applied to data taken with monochromatic $(\Delta E / E=0.014 \%) \mathrm{x}$ rays and polychromatic $(\Delta E / E=5 \%) \mathrm{x}$ rays, respectively.

From this figure one can conclude that the algorithm performs well for both the monochromatic and polychromatic settings. The reconstruction obtained using pink beam [Fig. 4(c)] shows even better contrast: the reasons for this are mainly due to the increased signal-to-noise ratio and better beam stability, resulting in an improved flat field correction.

In summary, the original algorithm works well in the case of strictly pure phase objects $(\beta=0)$ when one single data set acquired in the near field Fresnel region is sufficient (as demonstrated in the simulations).

This condition, however, is hard to realize experimentally because $\beta$ is never equal to zero in the considered wavelength range. Therefore, two tomographic data sets, one in the contact plane $(z=0)$ and one in the Fresnel region $(z$ $=d$ ), are required.

Applying additional high-pass filtering (MBA) to reduce the low frequency part of the noise to one single tomographic data set $(z=d)$ was sufficient to deliver quantitative information of index of refraction within few percent from tabulated data if a calibration to values of a known material is provided.

Good performance of the method when pink beam $(5 \%$ bandwidth) is used may allow faster measurements and potential applications with conventional sources. Future developments will focus on the fine tuning of the filtering step and on the streamlining of the whole process in order to allow high-resolution and high throughput imaging of soft biological specimens.

The authors are grateful to A. Bronnikov and F. Pfeiffer for fruitful discussions. Part of this work was supported by the Swiss National Science Foundation (FP-620-58097.99 and PP-104317/1).

${ }^{1}$ U. Bonse and F. Bush, Prog. Biophys. Mol. Biol. 65, 133 (1996).

${ }^{2}$ A. Momose, Nucl. Instrum. Methods Phys. Res. A 352, 622 (1995).

${ }^{3}$ T. Weitkamp, A. Diaz, C. David, F. Pfeiffer, M. Stampanoni, P. Cloetens, and E. Ziegler, Opt. Express 13, 6296 (2005).

${ }^{4}$ L. J. Allen, W. McBride, N. L. O'Leary, and M. P. Oxley, Ultramicroscopy 100, 91 (2004).

${ }^{5}$ W. K. Hsieh, F. R. Chen, J. J. Kai, and A. I. Kirkland, Ultramicroscopy 98, 99 (2004).

${ }^{6}$ S. C. Mayo, P. R. Miller, S. W. Wilkins, T. J. Davis, D. Gao, T. E. Gureyev, D. Paganin, D. J. Parry, A. Pogany, and A. W. Stevenson, J. Microsc. 207, 79 (2002).

${ }^{7}$ T. E. Gureyev, A. Pogany, D. M. Paganin, and S. W. Wilkins, Opt. Commun. 231, 53 (2004).

${ }^{8}$ A. G. Peele, F. DeCarlo, P. J. Mahon, B. B. Dhal, and K. A. Nugent, Rev. Sci. Instrum. 76, 083707 (2005).

${ }^{9}$ T. E. Gureyev and K. A. Nugent, J. Epidemiol. 13, 1670 (1996).

${ }^{10}$ P. Cloetens, W. Ludwig, J. Baruchel, D. Van Dyck, J. Van Landuyt, J. P. Guigay, and M. Schlenker, Appl. Phys. Lett. 75, 2912 (1999).

${ }^{11}$ F. Beckmann, M. Bartscher, T. Biermann, F. Busch, and O. Günnewig, Proc. SPIE 3149, 108 (1997).

${ }^{12}$ A. V. Bronnikov, Opt. Commun. 171, 239 (1999).

${ }^{13}$ A. V. Bronnikov, J. Opt. Soc. Am. A 19, 472 (2002).

${ }^{14}$ J. M. Cowley, Diffraction Physics (North-Holland, Amsterdam, 1995).

${ }^{15}$ A. Pogany, D. Gao, and S. W. Wilkins, Rev. Sci. Instrum. 68, 2774 (1997).

${ }^{16}$ M. Stampanoni, G. Borchert, P. Wyss, R. Abela, B. Patterson, S. Hunt, D. Vermeulen, and P. Rüegsegger, Nucl. Instrum. Methods Phys. Res. A 491, 291 (2002).

${ }^{17}$ CXRO database: http://www-cxro.lbl.gov/optical/constants/getdb2.html. 